\title{
A FIXED POINT THEOREM FOR A CLASS OF DIFFERENTIABLE STABLE OPERATORS IN BANACH SPACES
}

VADIM AZHMYAKOV

Received 31 January 2005; Accepted 10 October 2005

We study Fréchet differentiable stable operators in real Banach spaces. We present the theory of linear and nonlinear stable operators in a systematic way and prove solvability theorems for operator equations with differentiable expanding operators. In addition, some relations to the theory of monotone operators in Hilbert spaces are discussed. Using the obtained solvability results, we formulate the corresponding fixed point theorem for a class of nonlinear expanding operators.

Copyright (c) 2006 Vadim Azhmyakov. This is an open access article distributed under the Creative Commons Attribution License, which permits unrestricted use, distribution, and reproduction in any medium, provided the original work is properly cited.

\section{Introduction}

The basic inspiration for studying stable and strongly stable operators in a real Banach space $X$ is the operator equation of the form

$$
A(x)=a, \quad a \in X,
$$

where $A: X \rightarrow X$ is a nonlinear operator. We consider a single-valued mapping $A$, whose domain of definition is $X$ and whose range $R(A)$ is contained in $X$. Throughout this paper, the terms mapping, function, and operator will be used synonymously. We start by recalling some basic concepts and preliminary results (see, e.g., [29]).

Definition 1.1. An operator $A: X \rightarrow X$ is called stable if

$$
\left\|A\left(x_{1}\right)-A\left(x_{2}\right)\right\| \geq g\left(\left\|x_{1}-x_{2}\right\|\right) \quad \forall x_{1}, x_{2} \in X,
$$

where $g: \mathbb{R}_{+} \rightarrow \mathbb{R}_{+}$is a strictly monotone increasing and continuous function with

$$
g(0)=0, \quad \lim _{t \rightarrow+\infty} g(t)=+\infty .
$$

The function $g(\cdot)$ is called a stabilizing function of the operator $A$.

Hindawi Publishing Corporation

Fixed Point Theory and Applications

Volume 2006, Article ID 92429, Pages 1-17

DOI 10.1155/FPTA/2006/92429 
Let $H$ be a real Hilbert space. By $\langle\cdot, \cdot\rangle$ we denote the inner product of $H$. The Hilbert space $H$ will be identified with the dual space $H^{*}$. It is easy to see that Definition 1.1 is closely related to the concept of a coercive operator (see, e.g., [9]). Evidently, a stable operator $B: H \rightarrow H$ is coercive.

Definition 1.2. An operator $B: H \rightarrow H$ is called strongly stable if there is a number $c>0$ such that

$$
\left|\left\langle B\left(h_{1}\right)-B\left(h_{2}\right), h_{1}-h_{2}\right\rangle\right| \geq c|| h_{1}-h_{2} \|^{2} \quad \forall h_{1}, h_{2} \in H .
$$

Definition 1.2 coincides with the definition of a strongly monotone operator (see, e.g., $[21,29])$. Moreover, a uniformly monotone operator $B: H \rightarrow H$ is also stable [29]. Let $B$ be a strongly stable operator in a real Hilbert space $H$. The Schwarz inequality implies that

$$
\left\|B\left(h_{1}\right)-B\left(h_{2}\right)\right\| \geq c|| h_{1}-h_{2} \| \quad \forall h_{1}, h_{2} \in H
$$

We now suggest the following concept.

Definition 1.3. An operator $A: X \rightarrow X$ is called expanding if there is a number $d>0$ such that

$$
\left\|A\left(x_{1}\right)-A\left(x_{2}\right)\right\| \geq d\left\|x_{1}-x_{2}\right\| \quad \forall x_{1}, x_{2} \in X
$$

The number $d$ is called a constant of expansion.

It is evident that an expanding operator $A$ is a stable operator with the stabilizing function $g(t)=d \cdot t, t \geq 0$. It should be mentioned that in the literature, alternative definitions of stable operators are based on other viewpoints. For example, the theory of weakly stable operators in connection with the general approach to estimations for solutions of a class of perturbed operator equations is comprehensively discussed in [4].

Let $A: X \rightarrow X$ be stable. Then, for each $a \in X$, the operator equation (1.1) has at most one solution $\hat{x}$. To prove this, suppose that $A\left(\hat{x}_{1}\right)=A\left(\hat{x}_{2}\right)=a$, where $\hat{x}_{1}, \hat{x}_{2} \in X$. This implies that $g\left(\left\|\hat{x}_{1}-\hat{x}_{2}\right\|\right)=0$, and hence $\hat{x}_{1}=\hat{x}_{2}$. Consequently, a stable operator $A$ is injective. Moreover, we have the continuous dependence of the solution on the righthand side of the equation $A(x)=a$. From Definition 1.1, it follows that the solution $\hat{x}$ of (1.1) is "stable" in the following sense: for each $\epsilon>0$, there exists a number $\delta(\epsilon)>0$ such that

$$
\left\|a_{1}-a_{2}\right\|<\delta(\epsilon)
$$

where $a_{1}, a_{2} \in R(A)$ always imply that $\left\|\hat{x}_{1}-\hat{x}_{2}\right\|<\epsilon$ for the corresponding solution $\hat{x}_{1}$, $\hat{x}_{2} \in X$ of the problems $A(x)=a_{1}$ and $A(x)=a_{2}$, respectively.

The stable, strongly stable, and expanding operators play an important role in the general theory of discretization methods and in optimization (see, e.g., [5, 19, 20, 25, 29]). The aim of this paper is to study a class of Fréchet differentiable stable operators and to prove a solvability theorem for nonlinear operator equations (1.1) with differentiable expanding operators. Moreover, we examine the corresponding linearization of (1.1). 
The paper is organized as follows. In Section 2, we present some examples of stable and expanding operators. Basic theoretical facts on stable operators are contained in Section 3. In Sections 4 and 5, we prove our main results, namely, the solvability theorems for a class of operator equations (1.1) and for the corresponding linearized equation. As a corollary of the general solvability results, we obtain a fixed point theorem for a family of Fréchet differentiable expanding operators in real Banach spaces.

\section{Some examples of stable operators}

In this section we give some examples of stable and strongly stable operators. First we consider the case in which $X$ is finite-dimensional. Assume that a continuously differentiable functions $\gamma_{1}: \mathbb{R} \rightarrow \mathbb{R}$ satisfies

$$
\frac{d \gamma_{1}(x)}{d x} \geq d \quad \forall x \in \mathbb{R}
$$

with $d>0$. It is easy to see that $\gamma_{1}(\cdot)$ is an expanding function. Assume that a function $\gamma_{2}: \mathbb{R} \rightarrow \mathbb{R}$ is strongly stable (strongly monotone). Clearly, this condition is equivalent to the following:

$$
\inf _{x_{1} \neq x_{2}} \frac{\gamma_{2}\left(x_{1}\right)-\gamma_{2}\left(x_{2}\right)}{x_{1}-x_{2}}>0 .
$$

We now examine the function $\gamma_{3}(x)=|x|^{q} x, x \in \mathbb{R}, q \in \mathbb{N}$. It is a matter of direct verification to prove that this function is stable.

Example 2.1. Let $B: H \rightarrow H$ be a monotone operator on a real Hilbert space $H$. We have

$$
\begin{aligned}
\|\left(h_{1}\right. & \left.+B\left(h_{1}\right)\right)-\left(h_{2}+B\left(h_{2}\right)\right) \|^{2} \\
& =\left\langle\left(h_{1}+B\left(h_{1}\right)\right)-\left(h_{2}+B\left(h_{2}\right)\right),\left(h_{1}+B\left(h_{1}\right)\right)-\left(h_{2}+B\left(h_{2}\right)\right)\right\rangle^{2} \\
& =\left\langle B\left(h_{1}\right)-B\left(h_{2}\right), h_{1}-h_{2}\right\rangle+\left\|h_{1}-h_{2}\right\|^{2}+\left\|B\left(h_{1}\right)-B\left(h_{2}\right)\right\|^{2} \\
& \geq\left\|h_{1}-h_{2}\right\|^{2}+\left\|B\left(h_{1}\right)-B\left(h_{2}\right)\right\|^{2}
\end{aligned}
$$

for all $h_{1}, h_{2} \in H$. Denote by $I$ the identity operator. Thus the operator $(I+B)$ is an expanding operator,

$$
\left\|(I+B)\left(h_{1}\right)-(I+B)\left(h_{2}\right)\right\| \geq\left\|h_{1}-h_{2}\right\|
$$

with the constant of expansion $d=1$.

Example 2.2. Let $\omega: \mathbb{R} \rightarrow \mathbb{R}$ be a continuously differentiable function such that

$$
\omega^{\prime}(x) \geq d \quad \forall x \in \mathbb{R}
$$


and $d>0$. By $\mathbb{C}([0,1], \mathbb{R})$ we denote the space of all continuous functions from $[0,1]$ into $\mathbb{R}$. We now introduce a so-called Nemyckii operator $\mathcal{N}: \mathbb{C}([0,1], \mathbb{R}) \rightarrow \mathbb{C}([0,1], \mathbb{R})$ given by

$$
\mathcal{N}(x(\cdot))(\tau):=\omega(x(\tau))
$$

where $x(\cdot) \in \mathbb{C}([0,1], \mathbb{R})$. This operator is of frequent use in optimization theory and applications $[2,10]$. By the mean value theorem, we have $\left|\omega\left(x_{1}\right)-\omega\left(x_{2}\right)\right| \geq d\left|x_{1}-x_{2}\right|$, and therefore

$$
\begin{aligned}
\left\|\mathcal{N}\left(x_{1}(\cdot)\right)-\mathcal{N}\left(x_{2}(\cdot)\right)\right\|_{\mathbb{C}([0,1], \mathbb{R})} \\
\quad=\max _{0 \leq \tau \leq 1}\left|\mathcal{N}\left(x_{1}(\cdot)\right)(\tau)-\mathcal{N}\left(x_{2}(\cdot)\right)(\tau)\right| \\
\quad=\max _{0 \leq \tau \leq 1}\left|\omega\left(x_{1}(\tau)\right)-\omega\left(x_{2}(\tau)\right)\right| \geq d \max _{0 \leq \tau \leq 1}\left|x_{1}(\tau)-x_{2}(\tau)\right| \\
\quad=d\left\|x_{1}(\cdot)-x_{2}(\cdot)\right\|_{\mathbb{C}([0,1], \mathbb{R}) .}
\end{aligned}
$$

Consequently, the Nemyckii operator $\mathcal{N}(\cdot)$ is an expanding operator. Note that the introduced Nemyckii operator is Fréchet differentiable [2].

Let $\Omega \subset \mathbb{R}^{r}$ be a bounded smooth domain, $r \in \mathbb{N}, r \geq 2$. By $\mathbb{W}_{p}^{l}(\Omega)$ we denote the standard real Sobolev spaces endowed with the usual norms [1]. Here, $0 \leq p \leq \infty$ and $l \in \mathbb{N}$. Moreover, we set $\mathbb{M}^{l}(\Omega):=\mathbb{W}_{2}^{l}(\Omega)$. Using the standard notation

$$
D^{\beta} \varphi:=\frac{\partial^{|\beta|}}{\partial \xi_{1} \cdots \xi_{r}}, \quad|\beta|=\beta_{1}+\cdots+\beta_{r}, \beta=\left(\beta_{1}, \ldots, \beta_{r}\right) \in \mathbb{N}^{r},
$$

we define the seminorm $|\cdot|$ on $\mathbb{\boxplus}^{l}(\Omega)$ (containing the derivatives of order $l$ ),

$$
|v(\cdot)|:=\left[\sum_{|\beta|<l}\left|\int_{\Omega} D^{\beta} v(\cdot)\right|^{2}+\sum_{|\beta|=l} \int_{\Omega}\left|D^{\beta} v(\cdot)\right|^{2}\right]^{1 / 2} .
$$

Let $\mathbb{H}_{0}^{1}(\Omega)$ be the space of all elements from $\mathbb{H}^{1}(\Omega)$ vanishing on the boundary $\partial \Omega$ of $\Omega$ in the usual sense of traces. It is common knowledge that $\mathbb{G}_{0}^{1}(\Omega)$ is a Hilbert space $[1,16,17]$.

Example 2.3. Consider the following mildly nonlinear Dirichlet problem:

$$
\begin{gathered}
-\nabla \cdot(\zeta(v) \nabla v)=\psi \quad \text { in } \Omega, \\
v=0 \quad \text { on } \partial \Omega,
\end{gathered}
$$

where $\psi(\cdot) \in \mathbb{H}_{0}^{1}(\Omega)$ and $\zeta(\cdot): \mathbb{R} \rightarrow \mathbb{R}$ is a bounded Lipschitz continuous twice continuously differentiable function such that $\zeta(\cdot) \geq \zeta_{0}=$ const $>0$ uniformly on $\mathbb{R}$. For every function $v(\cdot) \in \mathbb{Q}_{0}^{1}(\Omega)$, we may write the following Poincaré inequality $[6,16,17]$ :

$$
C_{\Omega}|| v(\cdot)|| \leq|v(\cdot)|,
$$


where $C_{\Omega} \in \mathbb{R}_{+}$is a constant. Here, $|\cdot|$ is the above-mentioned seminorm on $\mathbb{Q}^{l}(\Omega)$. We now set $X=\mathbb{H}_{0}^{1}(\Omega)$. The Hilbert space $\mathbb{H}_{0}^{1}(\Omega)$ will be identified with the dual space $\left(\mathbb{H}_{0}^{1}(\Omega)\right)^{*}$. It can be proved that the operator

$$
\mathscr{A}: X \longrightarrow X, \quad \mathscr{A}(v(\cdot)):=\nabla v(\cdot)
$$

is an expanding operator $[4,5]$.

If in addition to the above-mentioned properties we assume that the function $\zeta(\cdot)$ is monotone increasing, then the nonlinear operator

$$
v(\cdot) \longrightarrow \sqrt{\zeta(v(\cdot))} \mathscr{A}(v(\cdot))
$$

is also an expanding operator [5].

Note that the Poincaré inequality can also be expressed in the form

$$
\|v(\cdot)\|_{\mathbb{L}^{2}(\Omega)} \leq C_{\Omega}\|\nabla u(\cdot)\|_{\mathbb{L}^{2}(\Omega)},
$$

where $\mathbb{L}^{2}(\Omega)$ is the Lebesgue space of all square-integrable functions and $v(\cdot) \in \mathbb{U}^{1}(\Omega)$ are the functions with vanishing mean value over $\Omega$. In some problems, one can compute the constant of expansion $C_{\Omega}$. For instance, in the case of a convex domain $\Omega$ with diameter $\rho$, we have $C_{\Omega}=\rho / \pi$ (see [7]).

Example 2.4. Consider a real Hilbert space $H$. According to the Riesz theorem, we define the bijective linear mapping $\mathscr{R}: H \rightarrow H$ (Riesz operator) such that

$$
\left\langle\mathscr{R} h^{*}, h\right\rangle=\left\langle h^{*}, h\right\rangle
$$

for all $h^{*} \in H$, and $\left\|\mathscr{R} h^{*}\right\|=\left\|h^{*}\right\|$. It is evident that the introduced Riesz operator is stable. Since a Hilbert space is a strictly convex Banach space $[13,26]$, for every $h \in H$ there exists a unique element $\mathscr{g} h \in H$ such that

$$
\langle\mathscr{G} h, h\rangle=\|h\|^{2}=\|\mathscr{g} h\|^{2}
$$

(see, e.g., [26]). The dualizing operator $J: H \rightarrow H$, as it is called, is also stable. Moreover, it follows that $\mathscr{F}=\mathscr{R}^{-1}$. Note that the dualizing operator can also be defined in a real Banach space $X[24]$.

Recall that a linear operator $\mathscr{A}: X \rightarrow X$ is called a linear homeomorphism if

$$
\mathscr{A}: X \rightarrow R(\mathscr{A})
$$

is a homeomorphism, or equivalently, if there exist positive constants $m$ and $M$ such that

$$
m\|x\| \leq\|\mathscr{A} x\| \leq M\|x\|
$$

for each $x \in X$. This fact is an immediate consequence of the Banach open mapping theorem (see, e.g., [3]). Clearly, every linear homeomorphism is a stable operator. 
Example 2.5. We continue by considering a linear symmetric operator $\mathscr{B}: H \rightarrow H$, where $H$ is a real Hilbert space. Let $\lambda$ be an eigenvalue of $\mathscr{B}$. Evidently, a symmetric operator $\mathscr{B} \in L(H, H)$ has only real eigenvalues [23]. An eigenvalue $\lambda$ is called a regular value of $\mathscr{B}$ if $(\lambda I-\mathscr{B})^{-1}$ exists and is bounded. Here $I$ is the identity operator. It is well known that a number $\lambda \in \mathbb{R}$ is a regular value of a symmetric operator $\mathscr{B}$ if and only if $(\lambda I-\mathscr{B})$ is an expanding operator with the constant of expansion

$$
d=\frac{1}{\|\operatorname{Res}(\lambda, \mathscr{B})\|_{L(H, H)}},
$$

where $\operatorname{Res}(\lambda, \mathscr{B})$ is the resolvent (see, e.g., [5]).

We now assume that $\lambda \in \mathbb{R}$ is a regular value of the symmetric operator $\mathscr{B}$ and

$$
\|\operatorname{Res}(\lambda, \mathscr{B})\|_{L(H, H)}<1
$$

Then the operator $(\lambda I-\mathscr{B})$ is expanding with the above constant of expansion $d$. Hence

$$
\|\lambda I h\|+\|\mathscr{S} h\| \geq\|(\lambda I-\mathscr{B}) h\| \geq d\|h\|
$$

for every $h \in H$. We have $\|\mathscr{S} h\| \geq(d-1)\|h\|$ for all $h \in H$. Thus the considered operator $\mathscr{B}$ is also expanding.

In conclusion of this section, we consider an important class of linear expanding operators in a real Banach space $X$. Let $\mathscr{A}: X \rightarrow X$ be a linear continuous operator. For $\mathscr{A}$, there exists a unique determined linear continuous adjoint operator $\mathscr{A}^{*} \in L\left(X^{*}, X^{*}\right)$, where $X^{*}$ is a topological dual space of $X$. It is well known that the following properties are equivalent [23]:

(i) $R(\mathscr{A})=X$,

(ii) the adjoint operator $\mathscr{A}^{*}$ is expanding.

As it is obvious from the foregoing, the class of stable and strongly stable operators is broadly representative.

\section{Theoretical background}

This section is devoted to some analytical properties of differentiable stable operators in real Banach spaces. We recall the Fréchet differentiability concept. Let $A: X \rightarrow X$ and $x_{0} \in X$. If there is a continuous linear mapping $A^{\prime}\left(x_{0}\right): X \rightarrow X$ with the property

$$
\lim _{\|\Delta x\| \rightarrow \infty} \frac{\left\|A\left(x_{0}+\Delta x\right)-A\left(x_{0}\right)-A^{\prime}\left(x_{0}\right) \Delta x\right\|}{\|\Delta x\|}=0,
$$

then $A^{\prime}\left(x_{0}\right)$ is called the Fréchet derivative of $A$ at $x_{0}$ and the operator $A$ is called Fréchet differentiable at $x_{0}$. According to this definition, we obtain

$$
A\left(x_{0}+\Delta x\right)=A\left(x_{0}\right)+A^{\prime}\left(x_{0}\right) \Delta x+o(\|\Delta x\|)
$$


where the expression $o(\|\Delta x\|)$ of this Taylor series has the property

$$
\lim _{\|\Delta x\| \rightarrow 0} \frac{o(\|\Delta x\|)}{\|\Delta x\|}=0 .
$$

We now introduce the hyperstability concept.

Definition 3.1. A stable operator $A: X \rightarrow X$ is called hyperstable if there exists a strictly monotone increasing and continuous function $\tilde{g}: \mathbb{R}_{+} \rightarrow \mathbb{R}_{+}$with

$$
\tilde{g}(0)=0, \quad \lim _{t \rightarrow+\infty} \tilde{g}(t)=+\infty
$$

such that the stabilizing function $g(\cdot)$ of $A$ satisfies the inequality

$$
k g\left(\frac{t}{k}\right) \geq \tilde{g}(t) \quad \forall t, k \in \mathbb{R}_{+} .
$$

For example, we may choose a linear function $\tilde{g}(\cdot)$. It is evident that every expanding operator is hyperstable. Consider the function $g(t)=e^{t}-1, t \in \mathbb{R}_{+}$. Evidently, this function satisfies all conditions of a stabilizing function. Since

$$
e^{t / k}-1 \geq \frac{t}{k} \quad \forall t, k \in \mathbb{R}_{+},
$$

we have $k g(t / k) \geq \tilde{g}(t), t, k \in \mathbb{R}_{+}$for the function $\tilde{g}(t)=t$. The following lemma is an easy consequence of the hyperstability property.

Lemma 3.2. Let $A: X \rightarrow X$ be hyperstable and Fréchet differentiable at $x_{0} \in X$. Then the linear operator $A^{\prime}\left(x_{0}\right) \in L(X, X)$ is stable.

Proof. Evidently,

$$
\begin{gathered}
A\left(x_{0}+x\right)=A\left(x_{0}\right)+A^{\prime}\left(x_{0}\right) x+\alpha\left(x_{0}, x\right) \quad \forall x \in X, \\
\lim _{\|x\| \rightarrow 0} \frac{\left\|\alpha\left(x_{0}, x\right)\right\|}{\|x\|}=0 .
\end{gathered}
$$

Using the triangle inequality and Definition 1.1, we obtain

$$
\left\|\alpha\left(x_{0}, x\right)\right\|+\left\|A^{\prime}\left(x_{0}\right) x\right\| \geq\left\|A\left(x_{0}+x\right)-A\left(x_{0}\right)\right\| \geq g(\|x\|) .
$$

For every $\epsilon>0$, we choose $\delta(\epsilon)>0$ such that $\|x\|<\delta(\epsilon)$ implies that $\left\|\alpha\left(x_{0}, x\right)\right\| \leq \epsilon\|x\|$. Hence

$$
\left\|A^{\prime}\left(x_{0}\right) x\right\| \geq g(\|x\|)-\left\|\alpha\left(x_{0}, x\right)\right\| \geq g(\|x\|)-\epsilon\|x\| .
$$

The inequality (3.9) holds for every $x \in X$ with $\|x\|<\delta(\epsilon)$. Consider an element $\xi \in X$ with $\|\xi\| \geq \delta(\epsilon)$ and select a number $k \in \mathbb{R}$ such that $(1 / k)\|\xi\|<\delta(\epsilon)$. Let $\tilde{x}:=\xi / k$. Since the operator $A^{\prime}\left(x_{0}\right)$ is linear, we obtain

$$
\frac{1}{k}\left\|A^{\prime}\left(x_{0}\right) \xi\right\|=\left\|A^{\prime}\left(x_{0}\right) \tilde{x}\right\| \geq g(\|\tilde{x}\|)-\epsilon\|\tilde{x}\|=g\left(\frac{1}{k}\|\xi\|\right)-\epsilon \frac{1}{k}\|\xi\|,
$$


and $\left\|A^{\prime}\left(x_{0}\right) \xi\right\| \geq k g((1 / k)\|\xi\|)-\epsilon\|\xi\|$. Since the operator $A$ is hyperstable, we have

$$
\left\|A^{\prime}\left(x_{0}\right) \xi\right\| \geq \tilde{g}(\|\xi\|)-\epsilon\|\xi\| \quad \forall \xi \in X .
$$

The inequality (3.11) holds for an arbitrary $\epsilon>0$ and $\xi \in X$. We conclude that

$$
\left\|A^{\prime}\left(x_{0}\right) \xi\right\| \geq \tilde{g}(\|\xi\|) \quad \forall \xi \in X
$$

Thus the operator $A^{\prime}\left(x_{0}\right) \in L(X, X)$ is stable and $\tilde{g}(\cdot)$ is the corresponding stabilizing function.

In the same vein, we have the following observation.

Corollary 3.3. Let $A: X \rightarrow X$ be an expanding and Fréchet differentiable at $x_{0} \in X$,

$$
\left\|A\left(x_{1}\right)-A\left(x_{2}\right)\right\| \geq d\left\|x_{1}-x_{2}\right\| \quad \forall x_{1}, x_{2} \in X \text {. }
$$

Then $A^{\prime}\left(x_{0}\right)$ is expanding with the same constant $d>0$,

$$
\left\|A^{\prime}\left(x_{0}\right) x_{1}-A\left(x_{0}\right) x_{2}\right\| \geq d\left\|x_{1}-x_{2}\right\| \quad \forall x_{1}, x_{2} \in X .
$$

By the statement that a nonlinear operator $A: X \rightarrow X$ is continuous we mean that this operator is norm continuous. Moreover, in this paper we consider only norm-closed subsets of a real Banach space $X$.

Lemma 3.4. Let $A: X \rightarrow X$ be stable and continuous. Then the range $R(A)$ is a closed subset of $X$.

Proof. Consider a sequence $\left\{y_{s}\right\} \subset R(A), s \in \mathbb{N}$ such that

$$
\lim _{s \rightarrow \infty}\left\|y_{s}-\tilde{y}\right\|=0, \quad \tilde{y} \in X .
$$

We now examine the corresponding sequence $\left\{x_{s}\right\} \subset X$ such that

$$
A\left(x_{s}\right)=y_{s}, \quad s \in \mathbb{N} .
$$

From

$$
\left\|y_{i}-y_{j}\right\|=\left\|A\left(x_{i}\right)-A\left(x_{j}\right)\right\| \geq g\left(\left\|x_{i}-x_{j}\right\|\right), \quad y_{i}, y_{j} \in\left\{y_{n}\right\}, i, j \in \mathbb{N},
$$

it follows that $\lim _{i, j \rightarrow \infty} g\left(\left\|x_{i}-x_{j}\right\|\right)=0$, where $g(\cdot)$ is the stabilizing function. Since this function $g: \mathbb{R}_{+} \rightarrow \mathbb{R}_{+}$is a strictly monotone increasing function with $g(0)=0$, we see that $\left\{x_{s}\right\}$ is a Cauchy sequence. Hence

$$
\lim _{s \rightarrow \infty}\left\|x_{s}-\tilde{x}\right\|=0, \quad \tilde{x} \in X
$$

Since $A$ is continuous, we have $A(\tilde{x})=\tilde{y}$. The proof is complete.

Assume that the range $R(A)$ of a stable continuous operator $A$ is a convex set. Then $R(A)$ is also closed in the weak topology on $X[22]$. A set $Q \subset X$ is called norm bounded 
if there is a constant $C \in \mathbb{R}_{+}$such that $\|x\| \leq C$ for all $x \in Q$. It is common knowledge that a weakly closed, norm-bounded subset of a normed space is weakly compact in the weak topology. Thus a norm-bounded convex range $R(A)$ of a stable continuous operator $A: X \rightarrow X$ is weakly compact.

Our next result is an immediate consequence of Lemmas 3.2, 3.4, and of the Banach open mapping theorem (see, e.g., $[3,23])$.

Lemma 3.5. Let $A: X \rightarrow X$ be hyperstable and Fréchet differentiable at $x_{0} \in X$. Then the operator $\left(A^{\prime}\left(x_{0}\right)\right)^{-1}: R\left(A^{\prime}\left(x_{0}\right)\right) \subseteq X \rightarrow X$ is linear and continuous.

Proof. By Lemma 3.2, the operator $A^{\prime}\left(x_{0}\right)$ is stable. Clearly, this operator is an injection. Lemma 3.4 implies that the range $R\left(A^{\prime}\left(x_{0}\right)\right)$ is a closed subset of $X$. Moreover, $R\left(A^{\prime}\left(x_{0}\right)\right)$ is a linear subspace of $X$. This shows that $R\left(A^{\prime}\left(x_{0}\right)\right)$ is also a Banach space. By the Banach open mapping theorem, the operator $\left(A^{\prime}\left(x_{0}\right)\right)^{-1}$ is linear and continuous.

Recall the definition of a linear compact operator [23].

Definition 3.6. Let $V$ be an open unit ball of the Banach space $X$. An operator $\mathscr{A} \in L(X, X)$ is called compact if the set $\mathscr{A}(V)$ is relatively compact (i.e., the closure of the set $\mathscr{A}(V)$ is compact).

We now present the following well-known fact (see, e.g., [30]).

Theorem 3.7. Let $\mathscr{A} \in L(X, X)$ be compact and $\operatorname{dim} R(\mathscr{A})=\infty$. Then $\mathscr{A}$ is not from the class of expanding operators.

Using Theorem 3.7, we can prove our next result.

Lemma 3.8. Let $A: X \rightarrow X$ be expanding and Fréchet differentiable at $x_{0} \in X$. If

$$
\operatorname{dim} R\left(A^{\prime}\left(x_{0}\right)\right)=\infty,
$$

then $A^{\prime}\left(x_{0}\right)$ is a noncompact operator.

Proof. By Corollary 3.3, the operator $A^{\prime}\left(x_{0}\right) \in L(X, X)$ is also expanding. By Theorem 3.7, the operator $A^{\prime}\left(x_{0}\right)$ is noncompact.

For a sequence $\left\{\Psi_{s}\right\}, s \in \mathbb{N}$, of operators $\Psi_{s}: X \rightarrow X$, one can consider the uniform convergence and the pointwise convergence. In the next lemma, we deal with a sequence of stable continuous operators and with the uniform limit of this sequence.

Lemma 3.9. Let $\left\{A^{s}\right\}, s \in \mathbb{N}$, be a sequence of stable continuous operators

$$
A^{s}: X \longrightarrow X
$$

and let $\left\{g^{s}(\cdot)\right\}$ be a sequence of stabilizing functions conforming to $\left\{A^{s}\right\}$. Assume that

$$
\inf \left\{g^{s}(\cdot)\right\} \geq g(\cdot) \text {, }
$$

where $g: \mathbb{R}_{+} \rightarrow \mathbb{R}_{+}$is a strictly monotone increasing and continuous function with

$$
g(0)=0, \quad \lim _{t \rightarrow+\infty} g(t)=+\infty
$$


and $R\left(A^{s}\right)=X$, for all $s \in \mathbb{N}$. If the operator $A: X \rightarrow X$ is the uniform limit of $\left\{A^{s}\right\}$, then $A$ is a stable continuous operator and $R(A)=X$. The function $g(\cdot)$ is a stabilizing function of A.

Proof. Using the stability of the mapping $A^{s}$ and the triangle inequality, we obtain

$$
\left\|A^{s}\left(x_{1}\right)-A^{s}\left(x_{2}\right)-\left(A\left(x_{1}\right)-A\left(x_{2}\right)\right)\right\|+\left\|A\left(x_{1}\right)-A\left(x_{2}\right)\right\| \geq g^{s}\left(\left\|x_{1}-x_{2}\right\|\right) \quad \forall x_{1}, x_{2} \in X .
$$

The uniform convergence implies that

$$
\lim _{s \rightarrow \infty}\left\|\left(A^{s}\left(x_{1}\right)-A^{s}\left(x_{2}\right)\right)-\left(A\left(x_{1}\right)-A\left(x_{2}\right)\right)\right\|=0 \quad \forall x_{1}, x_{2} \in X .
$$

Since $\inf \left\{g^{s}(\cdot)\right\} \geq g(\cdot)$, we have

$$
\left\|A\left(x_{1}\right)-A\left(x_{2}\right)\right\|_{Y} \geq g\left(\left\|x_{1}-x_{2}\right\|\right) \quad \forall x_{1}, x_{2} \in X .
$$

In other words, the operator $A$ is stable and the function $g(\cdot)$ is the corresponding stabilizing function. Since the uniform limit of a sequence of continuous operators is continuous (see, e.g., [8]), the operator $A$ is continuous.

Every operator $A^{s}, s \in \mathbb{N}$, is a surjection (we have $R\left(A^{s}\right)=X$ ). Given an element $y \in X$, we consider a sequence $\left\{x_{s}\right\} \subset X$ such that

$$
A^{s}\left(x_{s}\right)=y \quad \forall s \in \mathbb{N} .
$$

We deduce from the uniform convergence of the sequence $\left\{A^{s}\right\}$ that for each $\epsilon>0$, there exists a number $N(\epsilon) \in \mathbb{N}$ such that $\left\|A^{s}\left(x_{s}\right)-A^{r}\left(x_{s}\right)\right\| \leq \epsilon$ for $s, r \geq N(\epsilon)$. This means that $\left\{A^{s}\right\}$ is a uniformly Cauchy sequence [8]. Hence

$$
\left\|y-A^{r}\left(x_{s}\right)\right\| \leq \epsilon
$$

for $s, r \geq N(\epsilon)$. From the triangle inequality, it follows that

$$
\left\|y-A\left(x_{s}\right)\right\| \leq \epsilon-\left\|A\left(x_{s}\right)-A^{r}\left(x_{s}\right)\right\|
$$

for $s, r \geq N(\epsilon)$. Using the uniform convergence of the sequence $\left\{A^{s}\right\}$, we obtain

$$
\lim _{s \rightarrow \infty}\left\|y-A\left(x_{s}\right)\right\|=0
$$

Thus, the element $y \in X$ is a limit of the sequence $\left\{A\left(x_{s}\right)\right\}$ in $R(A)$. The operator $A$ is stable and continuous. According to Lemma 3.4, the range $R(A)$ is a closed subset of $X$. Consequently, $y \in R(A)$. Since the element $y$ is chosen as an arbitrary element of $X$, we see that $R(A)=X$. This completes the proof of the lemma.

From Lemma 3.8, it follows that a broad class of linear expanding operators is noncompact. The solvability theory for operator equations with compact operators has been 
given adequate consideration in the literature (see, e.g., [9]). Therefore, it is important to obtain the solvability criteria for operator equations with linear expanding operators.

\section{Linear expanding operators}

Let us reformulate the general (Lemma 3.9) for the linear expanding operators.

Lemma 4.1. Let $\left\{\mathscr{A}^{s}\right\} s \in \mathbb{N}$ be a sequence of expanding operators $\mathscr{A}^{s} \in L(X, X)$,

$$
\left\|A^{s}\left(x_{1}\right)-A^{s}\left(x_{2}\right)\right\| \geq d^{s}\left\|x_{1}-x_{2}\right\| \quad \forall x_{1}, x_{2} \in X .
$$

Assume that $\inf \left\{d^{s}\right\} \geq d>0$. If $\lim _{s \rightarrow \infty}\left\|\mathscr{A}^{s}-\mathscr{A}\right\|_{L(X, X)}=0$, then $\mathscr{A}$ is also an expanding linear continuous operator

$$
\left\|A\left(x_{1}\right)-A\left(x_{2}\right)\right\| \geq d\left\|x_{1}-x_{2}\right\| \quad \forall x_{1}, x_{2} \in X
$$

and $R(\mathscr{A})=X$.

We now introduce the set $\widetilde{\mathrm{x}}(X, X) \subset L(X, X)$ of all linear continuous surjective invertible operators. Let $\mathrm{s}(X, X)$ be the subset of all expanding operators from $\widetilde{\mathrm{N}}(X, X)$. Consider an operator $\mathscr{A} \in \widetilde{\kappa}(X, X)$. Evidently, the inverse operator $\mathscr{A}^{-1}$ is linear and continuous $[11,12]$. We examine an operator $\mathscr{D} \in L(X, X)$ such that

$$
\|\mathscr{D}\|_{L(X, X)}<\frac{1}{\left\|\mathscr{A}^{-1}\right\|_{L(X, X)}}
$$

Then the operator $(\mathscr{A}+\mathscr{D})$ is invertible and $(\mathscr{A}+\mathscr{D})^{-1}$ is linear and continuous (see $[11,12])$, that is, $(\mathscr{A}+\mathscr{D}) \in \widetilde{\mathfrak{N}}(X, X)$. In effect the set $\widetilde{\kappa}(X, X)$ is an open (in the norm topology) subset of $L(X, X)$. Using this fact, Lemma 3.4, and the Banach open mapping theorem, we can prove the next result.

Theorem 4.2. Assume that $\mathscr{A} \in \mathrm{N}(X, X)$ and that $\left\|\mathscr{A} x_{1}-\mathscr{A} x_{2}\right\| \geq d\left\|x_{1}-x_{2}\right\|$. Consider an operator $\mathscr{D} \in L(X, X)$ such that

$$
\|\mathscr{D}\|_{L(X, X)}<\min \left\{\frac{1}{\left\|\mathscr{A}^{-1}\right\|_{L(X, X)}}, \eta\right\}
$$

where $0<\eta<d$. Then the operator $(\mathscr{A}+\mathscr{D})$ is expanding and invertible. Moreover, $(\mathscr{A}+$ $\mathscr{D})^{-1}$ is a linear and continuous operator, that is, $(\mathscr{A}+\mathscr{D}) \in \mathrm{N}(X, X)$.

Proof. The expanding operator $\mathscr{A}$ is an injection. Lemma 3.4 implies that the range $R(\mathscr{A})$ is a closed subset of $X$. Moreover, $R\left(A^{\prime}\left(x_{0}\right)\right)$ is a linear subspace of $X$. By the Banach open mapping theorem, the operator $\mathscr{A}^{-1}$ is linear and continuous. Therefore, $\mathscr{A} \in \widetilde{\mathrm{N}}(X, X)$. Consider an operator $\mathscr{D} \in L(X, X)$ such that

$$
\|\mathscr{D}\|_{L(X, X)}<\frac{1}{\left\|\mathscr{A}^{-1}\right\|_{L(X, X)}}, \quad\|\mathscr{D}\|_{L(X, X)}<\eta
$$


where $\eta<d$. From the above-mentioned result, it follows that $(\mathscr{A}+\mathscr{D}) \in \tilde{\mathrm{N}}(X, X)$. Let $x \in X$. By the definition of the operator norm $\|\cdot\|_{L(X, X)}$, we obtain

$$
\|(\mathscr{A}+\mathscr{D}) x\| \geq\|\mathscr{A} x\|-\|\mathscr{D}\|_{L(X, X)}\|x\| \geq d\|x\|-\eta\|x\|=(d-\eta)\|x\| .
$$

It follows that $(\mathscr{A}+\mathscr{D}) \in \mathrm{s}(X, X)$.

The proved Theorem 4.2 establishes, in fact, that the set $\mathrm{\kappa}(X, X)$ is an open (in the norm topology) set in $L(X, X)$. Let us recall the following useful concept.

Definition 4.3. An operator $A: X \rightarrow X$, where $X$ is a Banach space, is called graph-closed if the graph $G_{A}:=\{(x, A x) \in X \times X: x \in X\}$ is a closed set.

We now take a look at the linear variant of the operator equation (1.1),

$$
\mathscr{A}(x)=a, \quad a \in X,
$$

where $\mathscr{A}: X \rightarrow X$ is a linear operator. Let $R(\mathscr{A})^{\perp}$ be the generalized orthogonal complement to the set $R(\mathscr{A})$. It is well known that we have $R(\mathscr{A})=X$ if and only if the following two conditions are satisfied:

(i) $R(\mathscr{A})^{\perp}=\{0\}$,

(ii) $R(\mathscr{A})$ is closed.

Using this fact and the concept of graph-closed operators, one can prove the following solvability result for (4.7) (see [28]).

Theorem 4.4. Let $\mathscr{A}: X \rightarrow X$ be a linear expanding and graph-closed operator, where $X$ is a real Banach space. Suppose that $R(\mathscr{A})^{\perp}=\{0\}$. Then, for each $a \in X,(4.7)$ has a unique solution.

Sometimes the closedness of $G_{A}$ for an operator $A: X \rightarrow X$ characterizes the continuity of $A$. An important case presents the closed graph theorem (see, e.g., [3]). If the linear operator $\mathscr{A}: X \rightarrow X$ is continuous, we can apply Lemma 3.4.

Theorem 4.5. Let $X$ be a real Banach space and let $\mathscr{A}: X \rightarrow X$ be a linear expanding and continuous operator. Suppose that $R(\mathscr{A})^{\perp}=\{0\}$. Then, for each $a \in X$, (4.7) has a unique solution.

Proof. By Lemma 3.4, the set $R(\mathscr{A})$ is closed. The expanding operator $\mathscr{A}$ is injective. Since $R(\mathscr{A})^{\perp}=\{0\}$, we also have $R(\mathscr{A})=X$.

In relation to the considered topics, it is pertinent to present the well-known solvability result for strongly stable (strongly monotone) linear operator in Hilbert spaces, namely, the Lax-Milgram lemma [14].

Theorem 4.6. Let $\mathscr{B}: H \rightarrow H$ be a linear strongly stable and continuous operator on a real Hilbert space $H$. Then for each $b \in H$, the equation $\mathscr{B} h=b, h \in H$, has a unique solution.

For some other related theorems, see also $[27,29]$. We recall that the classic result of Minty [18] states that a monotone operator $\mathscr{B}: H \rightarrow H$ is maximal monotone if and only if $(I+B)$ is surjective. Note that in this case, $B$ is also maximal accretive and the operator $(I+B)$ is expanding (see Example 2.1). 
Let $A: X \rightarrow X$ be a nonlinear operator. We now require for $A$ the existence of Fréchet derivatives $A^{\prime}\left(x_{0}\right)$ at the point $x_{0} \in X$. It makes sense to compare the nonlinear equation (1.1) with its linearization (4.7) at the point $x_{0} \in X$, where $\mathscr{A}=A^{\prime}\left(x_{0}\right)$. Thus we deal with the following linearized equation:

$$
A^{\prime}\left(x_{0}\right) x=a, \quad a \in X .
$$

Note that linearization techniques have long been recognized as a powerful tool for studying and solving operator equations. We now prove our basic theorem for a class of differentiable linear expanding operators.

Theorem 4.7. Let $X$ be a real Banach space. Let

(i) an operator $A: X \rightarrow X$ be expanding and continuously Fréchet differentiable at every point $x \in X$;

(ii) $R\left(A^{\prime}(\tilde{x})\right)=X$ for a point $\tilde{x} \in X$.

Then the linearized problem (4.8) has a unique solution for every $x_{0} \in X$.

Proof. By Corollary 3.3, the mapping $A^{\prime}(x)$ is expanding at every point $x \in X$. Moreover, the constant of expansion $d>0$ for $A^{\prime}(x)$ is the same. Let

$$
T:=\left\{x \in X: R\left(A^{\prime}(x)\right)=X\right\} .
$$

Since $R\left(A^{\prime}(\tilde{x})\right)=X$ for a $\tilde{x} \in X$, we have $T \neq \varnothing$. We claim that the set $T$ is a closed subset of $X$. To see this, consider a convergent sequence $\left\{x_{s}\right\}, x_{s} \in T, s \in \mathbb{N}$, such that

$$
\lim _{s \rightarrow \infty}\left\|x_{s}-\bar{x}\right\|=0
$$

The mapping $A^{\prime}: X \rightarrow L(X, X)$ is continuous. Hence

$$
\lim _{s \rightarrow \infty}\left\|A^{\prime}\left(x_{s}\right)-A^{\prime}(\bar{x})\right\|_{\mathscr{L}(X, X)}=0 .
$$

By Lemma 4.1, the continuous operator $A^{\prime}(\bar{x})$ is expanding. By Lemma 3.4,

$$
R\left(A^{\prime}(\bar{x})\right)=X
$$

This proves that $\bar{x} \in T$.

We now consider the set $\mathrm{\aleph}(X, X) \subset L(X, X)$. Evidently, $T=\left(A^{\prime}\right)^{-1}(\aleph(X, X))$, where $A^{\prime}: X \rightarrow L(X, X)$. The set $\mathrm{\kappa}(X, X)$ is an open (in the usual norm topology) subset of $L(X, X)$ (see $[11,12])$. Since the mapping $A^{\prime}: X \rightarrow L(X, X)$ is continuous, the set $T$ is also an open set in $X$. Thus the considered set $T \neq \varnothing$ is closed and open. Hence, $T=X$ and we obtain

$$
R\left(A^{\prime}\left(x_{0}\right)\right)=X \quad \forall x_{0} \in X
$$

Since the linear expanding operator $A^{\prime}\left(x_{0}\right)$ is injective, $A^{\prime}\left(x_{0}\right)$ is bijective for every element $x_{0} \in X$. This completes the proof.

The presented Theorem 4.7 establishes solvability results for a class of linearized operator equations (4.8). This class is defined by points of linearization $x_{0} \in X$ for the initial nonlinear problem (1.1) with a differentiable expanding operator $A$. 


\section{The main theorem on differentiable expanding operators}

We now formulate and prove the following new inverse mapping theorem for nonlinear differentiable expanding operators.

Theorem 5.1. Let $X$ be a real Banach space and let

(i) $A: X \rightarrow X$ be an expanding and continuously Fréchet differentiable operator;

(ii) $R\left(A^{\prime}(\tilde{x})\right)=X$ for an element $\tilde{x} \in X$.

Then the given operator $A$ has an inverse $A^{-1}: X \rightarrow X$ and (1.1) has a unique solution for every $a \in X$.

Proof. Clearly, the expanding operator $A$ is injective. We claim that $R(A)=X$. To see this, we introduce the following set (see the proof of Theorem 4.7):

$$
T:=\left\{x \in X: R\left(A^{\prime}(x)\right)=X\right\} .
$$

Since $R\left(A^{\prime}(\tilde{x})\right)=X$ for a $\tilde{x} \in X$, we have $T \neq \varnothing$. Consider a convergent sequence

$$
\left\{x_{s}\right\}, \quad x_{s} \in T, s \in \mathbb{N},
$$

such that

$$
\lim _{s \rightarrow \infty}\left\|x_{s}-\bar{x}\right\|=0
$$

The mapping $A^{\prime}: X \rightarrow L(X, X)$ is continuous. Hence

$$
\lim _{s \rightarrow \infty}\left\|A^{\prime}\left(x_{s}\right)-A^{\prime}(\bar{x})\right\|_{L(X, X)}=0 .
$$

By Lemma 4.1, the continuous operator $A^{\prime}(\bar{x})$ is expanding. By Lemma 3.4,

$$
R\left(A^{\prime}(\bar{x})\right)=X .
$$

This proves that $\bar{x} \in T$ and the set $T$ is closed.

Consider the set $\mathrm{\aleph}(X, X) \subset L(X, X)$. Evidently, $T=\left(A^{\prime}\right)^{-1}(\aleph(X, X))$, where

$$
A^{\prime}: X \longrightarrow L(X, X) \text {. }
$$

The set $\mathrm{N}(X, X)$ is an open subset of $L(X, X)$ (see $[11,12])$. Since the introduced mapping $A^{\prime}: X \rightarrow L(X, X)$ is continuous, the set $T$ is an open set in $X$. Thus, the considered set $T \neq \varnothing$ is closed and open. Hence, $T=X$ and

$$
R\left(A^{\prime}\left(x_{0}\right)\right)=X \quad \forall x_{0} \in X .
$$

Since the linear expanding operator $A^{\prime}\left(x_{0}\right)$ is also injective, $A^{\prime}\left(x_{0}\right)$ is bijective for every $x_{0} \in X$. Moreover, $\left(A^{\prime}\left(x_{0}\right)\right)^{-1}: X \rightarrow X$ is linear and continuous [11, 12]. According to the inverse function theorem (see, e.g., [23]), the range $R(A)$ of the nonlinear operator $A$ is an open subset of $X$.

By Lemma 3.4, the range $R(A)$ of the stable continuous operator $A$ is closed in $X$. Hence $R(A)=X$. This means that the expanding operator $A$ is bijective and there exists the inverse mapping $A^{-1}: X \rightarrow X$. The proof is finished. 
In contrast to Theorem 4.7, the formulated Theorem 5.1 represents a solvability result for a class of initial nonlinear equations (1.1) with differentiable expanding operators $A$. The assumptions of Theorem 5.1 are analogous to the assumptions of Theorem 4.7. Thus, under the conditions of Theorem 5.1, we obtain not only the solvability result for the linearized operator equation (4.8), but also the solvability of the initial operator equation (1.1).

We can expect that the existence of an inverse mapping for a given nonlinear expanding mapping of $X$ into itself involves a specific fixed point theorem. As a corollary of our main Theorem 5.1, we obtain the following fixed point theorem for differentiable expanding operators in real Banach spaces.

Theorem 5.2. Let $X$ be real Banach spaces and let

(i) $A: X \rightarrow X$ be a continuously Fréchet differentiable expanding operator with a constant of expansion $d>1$,

(ii) $R\left(A^{\prime}(\tilde{x})\right)=X$ for a $\tilde{x} \in X$.

Then the mapping $A$ has a unique fixed point.

Proof. We must show that the operator $(A-I)$, where $I$ is the identity operator, satisfies all assumptions of Theorem 5.1. Since $A$ is expanding, we have

$$
\begin{aligned}
& \left\|A\left(x_{1}\right)-x_{1}-A\left(x_{2}\right)-x_{2}\right\| \\
& \quad \geq\left\|A\left(x_{1}\right)-A\left(x_{2}\right)\right\|-\left\|x_{1}-x_{2}\right\| \geq(d-1)\left\|x_{1}-x_{2}\right\| \quad \forall x_{1}, x_{2} \in X .
\end{aligned}
$$

Moreover, we have $d-1>0$. We can also show that $R\left(A^{\prime}(\tilde{x})-I\right)=X$ (see proof of Theorem 4.7). Theorem 5.1 implies the existence of the inverse operator $(A-I)^{-1}$ and the existence of the fixed point

$$
x^{\mathrm{fix}}=(A-I)^{-1}\left(0_{X}\right)
$$

for the mapping $A$, where $0_{X}$ is the zero-element of $X$. Since $(A-I)$ is bijective, we deduce the uniqueness of the fixed point $x^{\mathrm{fix}} \in X$.

Our next fixed point theorem can be formulated as a corollary of the basic (Theorem 4.7) for linear expanding operators.

Theorem 5.3. Let $X$ be real Banach spaces and let

(i) $A: X \rightarrow X$ be a continuously Fréchet differentiable expanding operator with the constant of expansion $d>1$,

(ii) $R\left(A^{\prime}(\tilde{x})\right)=X$ for $a \tilde{x} \in X$.

Then the mapping $A^{\prime}\left(x_{0}\right)$ has a unique fixed point for every $x_{0} \in X$.

The presented Theorem 5.3 can be proved in the same way as Theorem 5.2. We now establish a linkage between monotone operators in Hilbert spaces and operators from Theorem 5.1.

Theorem 5.4. Let $B: H \rightarrow H$ be a monotone and continuously Fréchet differentiable operator on the real Hilbert space $H$. Let $R\left(B^{\prime}(\tilde{h})\right)=H$ for a vector $\tilde{h} \in H$. Then $B$ is a maximal monotone and maximal accretive operator. 
Proof. The operator $(I+B)$ is expansive (see Example 2.1). We deduce that

$$
R\left(I+B^{\prime}(\tilde{h})\right)=H
$$

for a vector $\tilde{h} \in H$. It follows that all conditions of Theorem 5.1 are satisfied and the operator $(I+B)$ is surjective on $H$. According to the Minty theorem [18], the operator $B$ is maximal monotone and maximal accretive. The proof is complete.

Finally, note that the theory of differentiable expanding operators has a wide application in the general theory of discretization methods and in optimization. For the corresponding examples, see, for example, $[5,19,20,25]$.

\section{Concluding remarks}

In this paper, we present the theory of differentiable stable and expanding operators in real Banach and Hilbert spaces. It is not difficult to see that many of the results obtained here also hold in the corresponding spaces over $\mathbb{C}$. The next question concerns a possible weakening of the "strong" Fréchet differentiability assumptions in our main results, namely, in Theorems 4.7 and 5.1. What are the weakened differentiability conditions under which the statement of the above theorems is correct? A constructive investigation in this context can follow the directions of the nonlinear analysis. It is not unlikely to prove the analogs of the main results using the generalized derivatives and the suitable variants of the implicit function theorem.

Finally, note that the stability concept proposed for the single-valued operators can also be extended to the multifunctions in Banach spaces. The next interesting problem is the generalization of the main results for the stable operators to the multivalued case.

\section{Acknowledgment}

The author is grateful to Professor A. Kaplan (University of Trier) and to Professor J. Eichhorn (University of Greifswald) for their helpful comments and suggestions.

\section{References}

[1] R. A. Adams, Sobolev Spaces, Pure and Applied Mathematics, vol. 65, Academic Press, New York, 1973.

[2] V. M. Alekseev, V. M. Tichomirov, and S. V. Fomin, Optimal Control, Contemporary Soviet Mathematics, Plenum Press, New York, 1987.

[3] C. D. Aliprantis and K. C. Border, Infinite-Dimensional Analysis, 2nd ed., Springer, Berlin, 1999.

[4] L. Angermann, A posteriori error estimates for approximate solutions of nonlinear equations with weakly stable operators, Numerical Functional Analysis and Optimization 18 (1997), no. 5-6, 447-459.

[5] V. Azhmyakov, Stable Operators in Analysis and Optimization, Peter Lang, Berlin, 2005.

[6] C. Baiocchi and A. Capelo, Variational and Quasivariational Inequalities. Applications to Free Boundary Problems, A Wiley-Interscience Publication, John Wiley \& Sons, New York, 1984.

[7] M. Bebendorf, A note on the Poincaré inequality for convex domains, Zeitschrift für Analysis und ihre Anwendungen 22 (2003), no. 4, 751-756.

[8] S. K. Berberian, Fundamentals of Real Analysis, Universitext, Springer, New York, 1999. 
[9] H. Gajewski, K. Gröger, and K. Zacharias, Nichtlineare Operatorgleichungen und Operatordifferentialgleichungen, Mathematische Lehrbücher und Monographien, II. Abteilung, Mathematische Monographien, vol. 38, Akademie, Berlin, 1974.

[10] A. D. Ioffe and V. M. Tihomirov, Theory of Extremal Problems, Studies in Mathematics and Its Applications, vol. 6, North-Holland, Amsterdam, 1979.

[11] A. N. Kolmogorov and S. V. Fomin, Introductory Real Analysis, Prentice-Hall, New York, 1970, Revised English edition, translated from the Russian and edited by R. A. Silverman.

[12] _ Elements of the Theory of Functions and Functional Analysis, Nauka, Moscow, 1972.

[13] G. Köthe, Topological Vector Spaces I, Springer, Berlin, 1983.

[14] P. D. Lax and A. N. Milgram, Parabolic equations, Contributions to the Theory of Partial Differential Equations. Proceedings of the Conference on Partial Differential Equations (L. Bers, S. Bochner, and F. John, eds.), Annals of Mathematics Studies, no. 33, Princeton University Press, New York, 1954, pp. 167-190.

[15] J.-L. Lions and E. Magenes, Non-Homogeneous Boundary Value Problems and Applications. Vol. I, Die Grundlehren der mathematischen Wissenschaften, vol. 181, Springer, New York, 1972.

[16] _ Non-Homogeneous Boundary Value Problems and Applications. Vol. II., Die Grundlehren der mathematischen Wissenschaften, vol. 182, Springer, New York, 1972.

[17] _ Non-Homogeneous Boundary Value Problems and Applications. Vol. III, Die Grundlehren der mathematischen Wissenschaften, vol. 183, Springer, New York, 1973.

[18] G. J. Minty, Monotone (nonlinear) operators in Hilbert space, Duke Mathematical Journal 29 (1962), 341-346.

[19] W. V. Petryshyn, On the approximation-solvability of equations involving A-proper and psuedo-Aproper mappings, Bulletin of the American Mathematical Society 81 (1975), 223-312.

[20] Solvability of linear and quasilinear elliptic boundary value problems via the A-proper mapping theory, Numerical Functional Analysis and Optimization 2 (1980), no. 7-8, 591-635.

[21] R. R. Phelps, Convex Functions, Monotone Operators and Differentiability, Lecture Notes in Mathematics, vol. 1364, Springer, Berlin, 1993.

[22] A. P. Robertson and W. Robertson, Topological Vector Spaces, Cambridge University Press, Cambridge, 1966.

[23] W. Rudin, Functional Analysis, McGraw-Hill Series in Higher Mathematics, McGraw-Hill, New York, 1973.

[24] M. Ruzicka, Nichtlineare Funktionalanalysis, Springer, Berlin, 2004.

[25] H. J. Stetter, Analysis of Discretization Methods for Ordinary Differential Equations, Springer Tracts in Natural Philosophy, vol. 23, Springer, New York, 1973.

[26] W. Takahashi, Nonlinear Functional Analysis, Yokohama, Yokohama, 2000.

[27] E. H. Zarantonello, Solving functional equations by contractive averaging, Tech. Rep. 160, Mathematics Research Centre, University of Wisconsin, Madison, 1960.

[28] E. Zeidler, Nonlinear Functional Analysis and Its Applications. II/A. Linear Monotone Operators, Springer, New York, 1990.

[29] __ Nonlinear Functional Analysis and Its Applications. II/B. Nonlinear Monotone Operators, Springer, New York, 1990.

[30] __ Nonlinear Functional Analysis and Its Applications. III. Variational Methods and Optimization, Springer, New York, 1990.

Vadim Azhmyakov: Fakultät IV-Elektrotechnik und Informatik, Institut für Energie und Automatisierungstechnik, Technische Universität Berlin, Sekretariat EN 11, Einsteinufer 17, 10587 Berlin, Germany

E-mail address: azhmyakov@control.tu-berlin.de 